\title{
Doubly and Triply Periodic Waves Solutions for the KdV Equation*
}

\author{
Ying Huang, Dengguo Xu \\ Department of Mathematics, Chuxiong Normal University, Chuxiong, China \\ Email: huang11261001@163.cn
}

Received September 6, 2013; revised October 6, 2013; accepted October 13, 2013

Copyright (C) 2013 Ying Huang, Dengguo Xu. This is an open access article distributed under the Creative Commons Attribution License, which permits unrestricted use, distribution, and reproduction in any medium, provided the original work is properly cited.

\begin{abstract}
Based on the arbitrary constant solution, a series of explicit doubly periodic solutions and triply periodic solutions for the Korteweg-de Vries (KdV) equation are first constructed with the aid of the Darboux transformation method.
\end{abstract}

Keywords: KdV Equation; Doubly Periodic Solution; Triply Periodic Solution; Darboux Transformation

\section{Introduction}

The famous KdV equation

$$
u_{t}+6 u u_{x}+u_{x x x}=0
$$

is a shallow water wave equation early derived by Korteweg de and Vries, its first application was discovered in the study of collision-free hydro-magnetic waves in 1960. Subsequently, it has arisen in a number of physical contexts, such as stratified internal waves, ionacoustic waves, plasma physics, lattice dynamics and so on. Following the further studies of these physical problems, its exact solutions have attracted much attention and have been extensively studied [1-7]. However, in contrast to solitary wave solutions, the analytic periodic solutions represent only a small subclass of its known solutions, and multi-periodic solutions are scarce. It is always useful to seek more and various multi-periodic solutions for recovering interactions among some simple periodic waves in a nonlinear medium.

We know that the Darboux transformation method is the main method to construct exact multi-soliton solutions, and this method is scarcely used for solving multiperiodic solutions [8-10]. In the paper, not only explicit doubly periodic solutions are available, but also a group of explicit triply periodic solutions is obtained by means of the Darboux transformation method.

*This work was supported by the Chinese Natural Science Foundation Grant (11261001) and Yunnan Provincial Department of Education Research Foundation Grant (2012Y130).

\section{Doubly Periodic Solutions}

According to [11], the linear system

$$
\left\{\begin{array}{l}
\Phi_{x}=\left(\begin{array}{cc}
0 & 1 \\
\lambda-u & 0
\end{array}\right) \Phi, \\
\Phi_{t}=\left(\begin{array}{cc}
u_{x} & -(4 \lambda+2 u) \\
A & -u_{x}
\end{array}\right) \Phi,
\end{array}\right.
$$

is the Lax pair for Equation (1), with the Darboux matrix

$$
D(x, t, \lambda)=\left(\begin{array}{cc}
-\sigma_{i} & 1 \\
\lambda-\lambda_{i}+\sigma_{i}^{2} & -\sigma_{i}
\end{array}\right),
$$

where $A=-(4 \lambda+2 u)(\lambda-u)+u_{x x}, \lambda, \lambda_{i}(i=0,1,2)$ are the spectral parameters. The monograph [11] further points out, if $u_{i}$ is a known solution to Equation (1), then

$$
u_{i+1}=2 \lambda_{i}-u_{i}-2 \sigma_{i}^{2}
$$

becomes new solution generated from $u_{i}$, with

$$
\sigma_{i}=\frac{a_{21}^{(i)}\left(x, t, \lambda_{i}\right) \mu_{i}+a_{22}^{(i)}\left(x, t, \lambda_{i}\right) \gamma_{i}}{a_{11}^{(i)}\left(x, t, \lambda_{i}\right) \mu_{i}+a_{12}^{(i)}\left(x, t, \lambda_{i}\right) \gamma_{i}},
$$

where, $\mu_{i}$ and $\gamma_{i}$ are arbitrary constants, but $\mu_{i}^{2}+\gamma_{i}^{2} \neq 0$, and $\Phi_{i}(x, t, \lambda)=\left(a_{j k}^{(i)}(x, t, \lambda)\right)_{2 \times 2}$ is the fundamental solution matrix to the lax pair on $u_{i}$.

Only solving the fundamental solution matrix of the lax pair corresponding to constant solution $u_{0}$, it is possible to construct multi-periodic solutions to the $\mathrm{KdV}$ Equation (1). Substituting $u_{0}$ into the system (2) yields 


$$
\left\{\begin{array}{l}
\Phi_{x}=\left(\begin{array}{cc}
0 & 1 \\
\lambda-u_{0} & 0
\end{array}\right) \Phi, \\
\Phi_{t}=-\left(4 \lambda+2 u_{0}\right)\left(\begin{array}{cc}
0 & 1 \\
\lambda-u_{0} & 0
\end{array}\right) \Phi .
\end{array}\right.
$$

If setting $\xi=x-\left(4 \lambda+2 u_{0}\right) t$, then we can assert that both the system (6) and the following linear system

$$
\Phi_{\xi}=\left(\begin{array}{cc}
0 & 1 \\
\lambda-u_{0} & 0
\end{array}\right) \Phi
$$

have exactly the same solutions. Under the condition for $u_{0}>\lambda$, by the eigenvalue method, we obtain the complex-valued fundamental solution matrix to the above system

$$
\left(\begin{array}{cc}
\mathrm{e}^{i a \xi} & \mathrm{e}^{-i a \xi} \\
i a \mathrm{e}^{i a \xi} & -i a \mathrm{e}^{-i a \xi}
\end{array}\right),
$$

where $a=a(\lambda)=\sqrt{u_{0}-\lambda}$. Because the real and imaginary parts of a complex-valued solution are also solutions, we thus take

$$
\Phi_{0}(x, t, \lambda)=\left(\begin{array}{cc}
\cos \theta & \sin \theta \\
-a \sin \theta & a \cos \theta
\end{array}\right)
$$

as the fundamental solution matrix to the the system (6), where $\theta=\theta(\lambda)=a \xi$.

For simplicity, we setting

$a_{i}=a\left(\lambda_{i}\right), \theta_{i}=\theta\left(\lambda_{i}\right), \Gamma_{i j}=a_{i}^{2}-a_{2}^{j}=\lambda_{j}-\lambda_{i}$, $(i, j=0,1,2)$.

From (5), we have

$$
\sigma_{0}=a_{0} \frac{-\mu_{0} \sin \theta_{0}+\gamma_{0} \cos \theta_{0}}{\mu_{0} \cos \theta_{0}+\gamma_{0} \sin \theta_{0}},
$$

in the above formula, choosing $\mu_{0}=1, \gamma_{0}=0$ and $\mu_{0}=0, \gamma_{0}=1$, respectively, we get

$$
\sigma_{0 t}=-a_{0} \tan \theta_{0}
$$

and

$$
\sigma_{0 c}=a_{0} \cot \theta_{0},
$$

respectively, with (4), the periodic wave solutions

$$
u_{1-1}=u_{0}-2 a_{0}^{2} \sec ^{2} \theta_{0}
$$

and

are obtained.

$$
u_{1-2}=u_{0}-2 a_{0}^{2} \csc ^{2} \theta_{0}
$$

Now we construct the doubly periodic solutions generated from $u_{1}$, thanks to (4), we see that

$$
u_{2}=2 \lambda_{1}-\left(2 \lambda_{0}-u_{0}-2 \sigma_{0}^{2}\right)-2 \sigma_{1}^{2},
$$

we first give $\sigma_{1}$, then substitute $\sigma_{0}$ and $\sigma_{1}$ into (11). Again according to [11], we can obtain the fundamental solution matrix to the lax pair associated with the known periodic wave solution $u_{1}$ in the following manner

$$
\begin{aligned}
\Phi_{1}(x, t, \lambda) & =\left(\begin{array}{cc}
-\sigma_{0} & 1 \\
\lambda-\lambda_{0}+\sigma_{0}^{2} & -\sigma_{0}
\end{array}\right) \Phi_{0}(x, t, \lambda) \\
& =\left(\begin{array}{cc}
-\sigma_{0} \cos \theta-a \sin \theta & -\sigma_{0} \sin \theta+a \cos \theta \\
P & Q
\end{array}\right),
\end{aligned}
$$

where $P=\left(\lambda-\lambda_{0}+\sigma_{0}^{2}\right) \cos \theta+\sigma_{0} a \sin \theta$, $Q=\left(\lambda-\lambda_{0}+\sigma_{0}^{2}\right) \sin \theta-\sigma_{0} a \cos \theta$. After combining (5) and (12), choosing $\mu_{1}=1, \gamma_{1}=0$, we get

$$
\sigma_{1 t}=-\frac{\left(\lambda_{1}-\lambda_{0}+\sigma_{0}^{2}\right)+\sigma_{0} a_{1} \tan \theta_{1}}{\sigma_{0}+a_{1} \tan \theta_{1}} .
$$

Substituting (9) and (13) into (11), we have new doubly periodic solution

$$
u_{2-1}=u_{0}+\frac{2 \Gamma_{01}\left(a_{0}^{2} \sec ^{2} \theta_{0}-a_{1}^{2} \sec ^{2} \theta_{1}\right)}{\left(a_{0} \tan \theta_{0}-a_{1} \tan \theta_{1}\right)^{2}} .
$$

Again substituting (10) and (13) into (11), we obtain another new doubly periodic solution

$$
u_{2-2}=u_{0}+\frac{2 \Gamma_{01}\left(a_{0}^{2} \csc ^{2} \theta_{0}-a_{1}^{2} \sec ^{2} \theta_{1}\right)}{\left(a_{0} \cot \theta_{0}+a_{1} \tan \theta_{1}\right)^{2}} .
$$

Similarly, choosing $\mu_{1}=0, \gamma_{1}=1$, we have

$$
\sigma_{1 c}=-\frac{\left(\lambda_{1}-\lambda_{0}+\sigma_{0}^{2}\right)-\sigma_{0} a_{1} \cot \theta_{1}}{\sigma_{0}-a_{1} \cot \theta_{1}},
$$

which implies the doubly periodic solutions

$$
u_{2-3}=u_{0}+\frac{2 \Gamma_{01}\left(a_{0}^{2} \sec ^{2} \theta_{0}-a_{1}^{2} \csc ^{2} \theta_{1}\right)}{\left(a_{0} \tan \theta_{0}+a_{1} \cot \theta_{1}\right)^{2}}
$$

and

$$
u_{2-4}=u_{0}+\frac{2 \Gamma_{01}\left(a_{0}^{2} \csc ^{2} \theta_{0}-a_{0}^{2} \csc ^{2} \theta_{1}\right)}{\left(a_{0} \cot \theta_{0}-a_{1} \cot \theta_{1}\right)^{2}} .
$$

Specially, although $u_{2-3}$ is a doubly periodic solution, its structure is very similar to a given two-soliton solution in [1].

\section{Triply Periodic Solutions}

As shown in [11], the fundamental solution matrix to the lax pair associated with the doubly periodic wave solution $u_{2}$ can be given by

$$
\Phi_{2}(x, t, \lambda)=\left(\begin{array}{cc}
-\sigma_{1} & 1 \\
\lambda-\lambda_{1}+\sigma_{1}^{2} & -\sigma_{1}
\end{array}\right) \Phi_{1}(x, t, \lambda),
$$

substituting (12) into (19), in exactly the same manner as in Section 2, we get 


$$
\sigma_{2 t}=\frac{\left(\lambda_{1}-\lambda_{2}\right)\left(\sigma_{0}+a_{2} \tan \theta_{2}\right)}{\lambda_{2}-\lambda_{0}+\left(\sigma_{0}+\sigma_{1}\right)\left(\sigma_{0}+a_{2} \tan \theta_{2}\right)}-\sigma_{1}
$$

and

$$
\sigma_{2 c}=\frac{\left(\lambda_{1}-\lambda_{2}\right)\left(\sigma_{0}-a_{2} \cot \theta_{2}\right)}{\lambda_{2}-\lambda_{0}+\left(\sigma_{0}+\sigma_{1}\right)\left(\sigma_{0}-a_{2} \cot \theta_{2}\right)}-\sigma_{1} .
$$

Owing to (4) and (11), we have

$$
u_{3}=2\left(\lambda_{0}-u_{0}-\sigma_{0}^{2}\right)+2\left(\lambda_{2}-\lambda_{1}+\sigma_{1}^{2}-\sigma_{2}^{2}\right)+u_{0} .
$$

Here, we set $F_{i}=a_{i} \tan \theta_{i}, G_{i}=a_{i} \cot \theta_{i}, i=0,1,2$. Substituting $\sigma_{0 t}, \sigma_{1 t}$ and $\sigma_{2 t}$ into (20), we obtain triply periodic solution

$$
\begin{aligned}
u_{3-1}= & u_{0}+2 a_{0}^{2} \sec ^{2} \theta_{0} \\
& +\frac{2 \Gamma_{12} \Gamma_{01}\left(a_{0}^{2} \sec ^{2} \theta_{0}-a_{1}^{2} \sec ^{2} \theta_{1}\right)\left(F_{0}-F_{2}\right)^{2}}{\left[\Gamma_{02}\left(F_{1}-F_{0}\right)+\Gamma_{10}\left(F_{0}-F_{2}\right)\right]^{2}} \\
& +\frac{2 \Gamma_{12} \Gamma_{02}\left(a_{2}^{2} \sec ^{2} \theta_{2}-a_{0}^{2} \sec ^{2} \theta_{0}\right)\left(F_{1}-F_{0}\right)^{2}}{\left[\Gamma_{02}\left(F_{1}-F_{0}\right)+\Gamma_{10}\left(F_{0}-F_{2}\right)\right]^{2}} .
\end{aligned}
$$

Similarly, we have

$$
\begin{aligned}
u_{3-2}= & u_{0}+2 a_{0}^{2} \csc ^{2} \theta_{0} \\
& +\frac{2 \Gamma_{12} \Gamma_{01}\left(a_{0}^{2} \csc ^{2} \theta_{0}-a_{1}^{2} \sec ^{2} \theta_{1}\right)\left(G_{0}+F_{2}\right)^{2}}{\left[\Gamma_{02}\left(F_{1}+G_{0}\right)-\Gamma_{10}\left(G_{0}+F_{2}\right)\right]^{2}} \\
& +\frac{2 \Gamma_{12} \Gamma_{02}\left(a_{2}^{2} \sec ^{2} \theta_{2}-a_{0}^{2} \csc ^{2} \theta_{0}\right)\left(F_{1}+G_{0}\right)^{2}}{\left[\Gamma_{02}\left(F_{1}+G_{0}\right)-\Gamma_{10}\left(G_{0}+F_{2}\right)\right]^{2}}, \\
u_{3-3}= & u_{0}+2 a_{0}^{2} \sec ^{2} \theta_{0} \\
& +\frac{2 \Gamma_{12} \Gamma_{01}\left(a_{0}^{2} \sec ^{2} \theta_{0}-a_{1}^{2} \csc ^{2} \theta_{1}\right)\left(F_{0}-F_{2}\right)^{2}}{\left[\Gamma_{02}\left(G_{1}+F_{0}\right)-\Gamma_{10}\left(F_{0}-F_{2}\right)\right]^{2}} \\
& +\frac{2 \Gamma_{12} \Gamma_{02}\left(a_{2}^{2} \sec ^{2} \theta_{2}-a_{0}^{2} \sec ^{2} \theta_{0}\right)\left(G_{1}+F_{0}\right)^{2}}{\left[\Gamma_{02}\left(G_{1}+F_{0}\right)-\Gamma_{10}\left(F_{0}-F_{2}\right)\right]^{2}}, \\
u_{3-4}= & u_{0}+2 a_{0}^{2} \csc ^{2} \theta_{0} \\
& +\frac{2 \Gamma_{12} \Gamma_{01}\left(a_{0}^{2} \csc ^{2} \theta_{0}-a_{1}^{2} \csc ^{2} \theta_{1}\right)\left(G_{0}+F_{2}\right)^{2}}{\left[\Gamma_{02}\left(G_{1}-G_{0}\right)+\Gamma_{10}\left(G_{0}+F_{2}\right)\right]^{2}} \\
& +\frac{2 \Gamma_{12} \Gamma_{02}\left(a_{2}^{2} \sec ^{2} \theta_{2}-a_{0}^{2} \csc ^{2} \theta_{0}\right)\left(G_{1}-G_{0}\right)^{2}}{\left[\Gamma_{02}\left(G_{1}-G_{0}\right)+\Gamma_{10}\left(G_{0}+F_{2}\right)\right]^{2}},
\end{aligned}
$$

$$
\begin{aligned}
u_{3-5}= & u_{0}+2 a_{0}^{2} \sec ^{2} \theta_{0} \\
& +\frac{2 \Gamma_{12} \Gamma_{01}\left(a_{0}^{2} \sec ^{2} \theta_{0}-a_{1}^{2} \sec ^{2} \theta_{1}\right)\left(F_{0}+G_{2}\right)^{2}}{\left[\Gamma_{02}\left(F_{1}-F_{0}\right)+\Gamma_{10}\left(F_{0}+G_{2}\right)\right]^{2}} \\
& +\frac{2 \Gamma_{12} \Gamma_{02}\left(a_{2}^{2} \csc ^{2} \theta_{2}-a_{0}^{2} \sec ^{2} \theta_{0}\right)\left(F_{1}-F_{0}\right)^{2}}{\left[\Gamma_{02}\left(F_{1}-F_{0}\right)+\Gamma_{10}\left(F_{0}+G_{2}\right)\right]^{2}}, \\
u_{3-6}= & u_{0}+2 a_{0}^{2} \csc ^{2} \theta_{0} \\
& +\frac{2 \Gamma_{12} \Gamma_{01}\left(a_{0}^{2} \csc ^{2} \theta_{0}-a_{1}^{2} \sec ^{2} \theta_{1}\right)\left(G_{0}-G_{2}\right)^{2}}{\left[\Gamma_{02}\left(F_{1}+G_{0}\right)-\Gamma_{10}\left(G_{0}-G_{2}\right)\right]^{2}} \\
& +\frac{2 \Gamma_{12} \Gamma_{02}\left(a_{2}^{2} \csc ^{2} \theta_{2}-a_{0}^{2} \csc ^{2} \theta_{0}\right)\left(F_{1}+G_{0}\right)^{2}}{\left[\Gamma_{02}\left(F_{1}+G_{0}\right)-\Gamma_{10}\left(G_{0}-G_{2}\right)\right]^{2}}, \\
u_{3-7}= & u_{0}+2 a_{0}^{2} \sec ^{2} \theta_{0} \\
& +\frac{2 \Gamma_{12} \Gamma_{01}\left(a_{0}^{2} \sec ^{2} \theta_{0}-a_{1}^{2} \csc ^{2} \theta_{1}\right)\left(F_{0}+G_{2}\right)^{2}}{\left[\Gamma_{02}\left(G_{1}+F_{0}\right)-\Gamma_{10}\left(F_{0}+G_{2}\right)\right]^{2}} \\
& +\frac{2 \Gamma_{12} \Gamma_{02}\left(a_{2}^{2} \csc ^{2} \theta_{2}-a_{0}^{2} \sec ^{2} \theta_{0}\right)\left(G_{1}+F_{0}\right)^{2}}{\left[\Gamma_{02}\left(G_{1}+F_{0}\right)-\Gamma_{10}\left(F_{0}+G_{2}\right)\right]^{2}},
\end{aligned}
$$

and

$$
\begin{aligned}
u_{3-8}= & u_{0}+2 a_{0}^{2} \csc ^{2} \theta_{0} \\
& +\frac{2 \Gamma_{12} \Gamma_{01}\left(a_{0}^{2} \csc ^{2} \theta_{0}-a_{1}^{2} \csc ^{2} \theta_{1}\right)\left(G_{0}-G_{2}\right)^{2}}{\left[\Gamma_{02}\left(G_{1}-G_{0}\right)+\Gamma_{10}\left(G_{0}-G_{2}\right)\right]^{2}} \\
& +\frac{2 \Gamma_{12} \Gamma_{02}\left(a_{2}^{2} \csc ^{2} \theta_{2}-a_{0}^{2} \csc ^{2} \theta_{0}\right)\left(G_{1}-G_{0}\right)^{2}}{\left[\Gamma_{02}\left(G_{1}-G_{0}\right)+\Gamma_{10}\left(G_{0}-G_{2}\right)\right]^{2}} .
\end{aligned}
$$

\section{REFERENCES}

[1] M. J. Ablowitz and P. A. Clarkson, "Solitons, Nonlinear Evolutions and Inverse Scattering," Cambridge University Press, Cambridge, 1991, pp. 23-99. http://dx.doi.org/10.1017/CBO9780511623998

[2] J. Álvarez and A. Durán, "Error Propagation When Approximating Multi-Solitons: The KdV Equation with as a Case Study," Applied Mathematics and Computation, Vol. 217, No. 4, 2010, pp. 1522-1539. http://dx.doi.org/10.1016/j.amc.2009.06.033

[3] J. L. Yin and L. X. Tian, "Classification of the Traveling Waves in the Nonlinear Dispersive KdV Equation,” Nonlinear Analysis, Vol. 73, No. 2, 2010, pp. 465-470. http://dx.doi.org/10.1016/j.na.2010.03.039

[4] A. Biswas, M. D. Petkovic and D. Milovic, "Topological and Non-Topological Exact Soliton Solution of the KdV Equation,” Nonlinear Science and Numerical Simulation, Vol. 15, No. 11, 2010, pp. 3263-3269. http://dx.doi.org/10.1016/j.na.2010.03.039 
[5] M. Nivala and B. Deconinck, "Periodic Finite-Genus Solutions of the KdV Equation Are Orbitally Stable," Physica D: Nonlinear Phenomena, Vol. 239, No. 13, 2011, pp. 1147-1158.

http://dx.doi.org/10.1016/j.physd.2010.03.005

[6] Y. Yamamoto, T. Nagase and M. Ohmiya, “Appell's Lemma and Conservation Laws of KdV Equation,” Journal of Computational and Applied Mathematics, Vol. 233, No. 6, 2010, pp. 1612-1618. http://dx.doi.org/10.1016/j.cam.2009.02.076

[7] N. K. Ameine and M. A. Ramadau, "A Small Time Solutions for the KdV Equation Using Bubnov-Galerkin Finite Element Method," Journal of the Egyptian Mathematical Society, Vol. 19, No. 3, 2011, pp. 118-125. http://dx.doi.org/10.1016/j.joems.2011.10.005

[8] X. M. Li and A. H. Chen, "Darboux Transformation and Multi-Soliton Solutions of Boussinesq-Burgers Equa- tion,” Physics Letters A, Vol. 342, No. 5-6, 2005, pp. 413-420. http://dx.doi.org/10.1016/j.physleta.2005.05.083

[9] Y. Wang, L. J. Shen and D. L. Du, "Darboux Transformation and Explicit Solutions for Some $(2+1)$-dimensional Equation,” Physics Letters A, Vol. 366, No. 3, 2007, pp. 230-240.

http://dx.doi.org/10.1016/j.physleta.2007.02.043

[10] H. X. Wu, Y. B. Zeng and T. Y. Fan, "Complexitons of the Modified KdV Equation by Darboux Transformation," Applied Mathematics and Computation, Vol. 196, No. 2, 2008, pp. 501-510. http://dx.doi.org/10.1016/j.amc.2007.06.011

[11] C. H. Gu, H. S. Hu and Z. X. Zhou, "Darboux Transformation in Soliton Theory and Its Applications on Geometry," Shanghai Scientific and Technical Publishers, Shanghai, 2005. 